\title{
Uso do Azul de Metileno no Tratamento de Choque Anafilático durante Anestesia. Relato de Caso *
}

\section{Methylene Blue to Treat Anaphylaxis during Anesthesia. Case Report}

Renato Mestriner Stocche, TSA ${ }^{1}$; Luís Vicente Garcia, TSA ${ }^{2}$; Marlene Paulino dos Reis, TSA ${ }^{3}$; Jyrson Guilherme Klamt, TSA ${ }^{4}$; Paulo Roberto B. Évora ${ }^{5}$

\section{RESUMO}

Stocche RM, Garcia LV, Reis MP, Klamt JG, Evora PRB - Uso do Azul de Metileno no Tratamento de Choque Anafilático durante Anestesia. Relato de Caso

JUSTIFICATIVA E OBJETIVOS: No período peri-operatório, o risco de anafilaxia deve sempre ser considerado. A incidência de reações alérgicas em anestesia é controversa, variando entre $1 / 3000$ a $1 / 20.000$, com mortalidade entre $3 \%$ e $9 \%$. Neste caso, relata-se o uso do azul de metileno como coadjuvante ao tratamento do choque anafilático refratário à terapêutica tradicional.

RELATO DO CASO: Paciente do sexo masculino, 53 anos, submetido a herniorrafia inguinal sob raquianestesia. No final do procedimento, ao receber dipirona $(1,5 \mathrm{~g})$, por via venosa, o paciente imediatamente apresentou broncoespasmo, cianose, diminuição da $\mathrm{SpO}_{2}$ e da PAS, culminando com parada cardiorrespiratória. Foi iniciada reanimação cardiorrespiratória com massagem cardíaca externa, seguida de IOT e injeção de adrenalina $(1 \mathrm{mg})$, atropina $(1 \mathrm{mg})$, restabelecendo-se FC de 150 bpm, porém sem pulso palpável. Administrou-se mais $1 \mathrm{mg}$ de adrenalina além de $1 \mathrm{~g}$ de hidrocortisona, com restabelecimento de pulso central (8 minutos). Apesar de receber dopamina (20 $\left.\mu \mathrm{g} \cdot \mathrm{kg}^{-1} \cdot \mathrm{min}^{-1}\right)$, o paciente manteve-se hipotenso $(60 \mathrm{mmHg})$ até 80 minutos. Administraram-se $100 \mathrm{mg}$ de azul de metileno por via venosa, quando houve aumento da PAS para 85 e $105 \mathrm{mmHg}$, após a segunda dose. Seguiu-se da diminuição da dose de dopamina de 20 para $10,7,5$ e, finalmente, $2 \mu \mathrm{g} \cdot \mathrm{kg}^{-1} \cdot \mathrm{min}^{-1}$.

CONCLUSÕES: A anafilaxia tem como principal mediador a liberação de histamina, que induz a produção de óxido nítrico (NO), com conseqüente aumento da guanilato ciclase que promove vasodilatação arteriolar por aumento do GMP cíclico. O azul de metileno pode ser útil nestas situações, pois inibe a guanilato ciclase e conseqüentemente a vasodilatação, o que resulta em melhora hemodinâmica.

Unitermos: ANALGÉSICOS, dipirona; COMPLICAÇÕES, choque; DROGAS, azul de metileno

* Recebido do (Received from) Serviço de Anestesiologia do Hospital das Clínicas de Ribeirão Preto, SP (HC - FMRP - USP)

1. Médico Assistente do Serviço de Anestesiologia do HC da FMRP - USP

2. Professor Assistente Doutor da Disciplina de Anestesiologia da FMRP USP

3. Professora Associada da Disciplina de Anestesiologia da FMRP - USP

4. Professor Assistente Doutor da Disciplina de Anestesiologia da FMRP USP

5. Médico Assistente do Departamento de Cirurgia do HC da FMRP - USP, Chefe do Laboratório Experimental de Óxido Nítrico da FMRP - USP

Apresentado (Submitted) em 08 de março de 2003

Aceito (Accepted) para publicação em 01 de julho de 2004

Endereço para correspondência (Correspondence to)

Dr. Renato Mestriner Stocche

Rua Adolfo Serra, 237 Alto da Boa Vista

14025-520 Ribeirão Preto, SP

E-mail: rstocche@keynet.com.br

(c) Sociedade Brasileira de Anestesiologia, 2004

\section{SUMMARY}

Stocche RM, Garcia LV, Reis MP, Klamt JG, Evora PRB - Methylene Blue to Treat Anaphylaxis during Anesthesia. Case Report

BACKGROUND AND OBJECTIVES: The risk of perioperative anaphylaxis should always be considered. The incidence of anesthetic allergic reactions is controversial, varying from 1/3,000 to $1 / 20,000$, with mortality range between 3 and $9 \%$. This report describes the use of methylene blue as coadjuvant drug to treat anaphylaxis refractory to conventional therapy.

CASE REPORT: A 53-year-old male patient was submitted to inguinal hernia correction under spinal anesthesia. After receiving $1.5 \mathrm{~g}$ intravenous dipirone at surgery completion, he immediately developed bronchospasm, cyanosis, decreased $\mathrm{SpO}_{2}$ and SBP, culminating with cardiac arrest. Resuscitation was started with external cardiac massage followed by tracheal intubation, as well as $1 \mathrm{mg}$ epinephrine and $1 \mathrm{mg}$ atropine injections. Heart rate returned $(150 \mathrm{bpm})$ with no palpable pulse though. Additional $1 \mathrm{mg}$ epinephrine and $1 \mathrm{~g}$ hydrocortisone were administered with central pulse recovery (8 minutes). Although receiving dopamine $\left(20 \mu \mathrm{g} \cdot \mathrm{kg}^{-1} \cdot \mathrm{min}^{-1}\right)$, patient remained hypotensive $(60 \mathrm{mmHg})$ until 80 minutes. Intravenous $100 \mathrm{mg}$ methylene blue was then administered with increased SBP to 85 and $105 \mathrm{mmHg}$ after the second dose. Dopamine dose was tapered from 10 to 7,5 and finally $2 \mu \mathrm{g} \cdot \mathrm{kg}^{-1} \cdot \mathrm{min}^{-1}$.

CONCLUSIONS: Histamine is the major anaphylaxis mediator. Inducing nitric oxide (NO) production, it consequently increases guanylate cyclase, which promotes arteriolar vasodilation by increasing cyclic GMP. Methylene blue may be helpful in such situations because it inhibits guanylate cyclase and consequently vasodilation, resulting in hemodynamic improvement.

Key Words: ANALGESICS dipirone; COMPLICATIONS, shock; DRUGS, methylene blue

\section{INTRODUÇÃO}

No período peri-operatório o risco de anafilaxia está aumentado, conseqüente à exposição a um grande número de fármacos. O primeiro relato de anafilaxia peri-operatória data de 1936, quando a paciente apresentou reação inesperada e intensa após a administração de D-tubocurarina ${ }^{1}$. A incidência de reações alérgicas em anestesia é controversa, variando entre $1 / 3000$ a $1 / 20000$ e, mesmo com tratamento clássico imediato (adrenalina, expansão volêmica, corticosteróides, suporte ventilatório e anti-histamínicos), a mortalidade pode ser alta, entre $3 \%$ e $9 \%{ }^{2-4}$.

A anafilaxia é uma reação complexa e parcialmente esclarecida. Há liberação de uma série de mediadores químicos responsáveis pelo quadro de colapso cardiovascular e respiratório. Dentre os principais mediadores encontram-se a histamina e a bradicinina ${ }^{5}$. Aliberação maciça destes mediadores 
induzem à produção de óxido nítrico ${ }^{5-8}$, que aumenta o GMPc no endotélio vascular, promovendo a característica vasodilatação arteriolar ${ }^{9}$.

Neste caso, relata-se o uso do azul de metileno, um inibidor da GMPc-sintase, como coadjuvante no tratamento de choque anafilático refratário à terapêutica tradicional.

\section{RELATO DO CASO}

Paciente do sexo masculino, 53 anos, submetido à herniorrafia inguinal direita sob raquianestesia com bupivacaína hiperbárica (15 mg), sedado com midazolam ( $3 \mathrm{mg}$ ) e monitorizado com cardioscopia $D_{\|}$e $V 5$, pressão arterial automática (método oscilométrico) e oximetria de pulso.

No final do procedimento, o paciente apresentava-se acordado e hemodinamicamente estável há mais de uma hora (PA de 125/80 mmHg), quando recebeu dipirona (1,5 g), por via venosa, para analgesia pós-operatória. Imediatamente, o paciente referiu dormência na língua, evoluindo com broncoespasmo e diminuição da pressão arterial sistólica (70 $\mathrm{mmHg}$ ). Foi administrado $\mathrm{O}_{2}$ a $100 \%$ sob máscara e injetado $10 \mathrm{mg}$ de efedrina. Porém, rapidamente seguiu-se piora do quadro respiratório, cianose, edema de glote, eritema cutâneo, diminuição da saturação da hemoglobina, pressão arterial não detectada pelo método oscilométrico, pulsos periféricos ausentes e diminuição da FC para $37 \mathrm{bpm}$.

Iniciaram-se as manobras de reanimação com administração, por via venosa, de adrenalina (1 mg), atropina (1 mg) e succinilcolina (100 mg), seguida de intubação traqueal, ventilação manual com oxigênio a $100 \%$ e massagem cardíaca externa. Seguiu-se o restabelecimento da atividade elétrica cardíaca sem pulso, quando foi administrado mais $1 \mathrm{mg}$ de adrenalina e $1 \mathrm{~g}$ hidrocortisona, sem melhora do quadro hemodinâmico. Já com 8 minutos, foi administrado mais $1 \mathrm{mg}$ de adrenalina, quando o paciente apresentou taquicardia ventricular sustentada, revertida com lidocaína $\left(2 \mathrm{mg} \cdot \mathrm{kg}^{-1}\right)$. Após a reversão da taquicardia ventricular sustentada (10 minutos), ocorreu melhora hemodinâmica (140 bpm e PAS de $60 \mathrm{mmHg}$ ). Iniciou-se, então, a administração de aminofilina e dopamina $\left(20 \mu \mathrm{g} \cdot \mathrm{kg}^{-1} \cdot \mathrm{min}^{-1}\right)$. Passados 25 minutos, houve alteração do ritmo cardíaco para fibrilação atrial com alta resposta ventricular e diminuição da PAS para $50 \mathrm{mmHg}$, que foi revertida na terceira tentativa de cardioversão (100, 150 e $200 \mathrm{~J}$ ). Nesse ínterim, procedeu-se sondagem vesical e nasogástrica, punção venosa central e solicitou-se gasometria arterial e dosagem sangüínea de eletrólitos. Com 80 minutos, já corrigida a volemia com $2500 \mathrm{ml}$ de cristalóides e $500 \mathrm{ml}$ de poliamida a $4 \%$ (PVC de $14 \mathrm{cmH}_{2} \mathrm{O}$ ) e sem distúrbios eletrolíticos, o paciente mantinha taquicardia sinusal (145 bpm) e hipotensão arterial grave (PAS entre 60 e $75 \mathrm{mmHg}$ ), mesmo recebendo altas doses de dopamina (20 $\left.\mu \mathrm{g} \cdot \mathrm{kg}^{-1} \cdot \mathrm{min}^{-1}\right)$.

Decidiu-se por administrar $100 \mathrm{mg}$ de azul de metileno, por via venosa, em injeção lenta (5 minutos). Imediatamente após a administração, a PAS aumentou para $85 \mathrm{mmHg}$. Seguiram-se mais 5 minutos mantendo esta discreta melhora, quando foi repetida a dose de $100 \mathrm{mg}$ de azul de metileno, com aumento subseqüente da PAS para $105 \mathrm{mmHg}$. Na seqüência, iniciou-se a diminuição rápida (20 minutos) da dose de dopamina de 20 para $10,7,5 \mathrm{e}$, finalmente, $2 \mu \mathrm{g} \cdot \mathrm{kg}^{-1} \cdot \mathrm{min}^{-1}$ que foi mantida para estimular a diurese.

Após 155 minutos do início do quadro, o paciente foi transferido para UTI recebendo dopamina $\left(2 \mu \mathrm{g} \cdot \mathrm{kg}^{-1} \cdot \mathrm{min}^{-1}\right)$, com boa diurese $\left(2 \mathrm{ml}_{\mathrm{kg}} \mathrm{kg}^{-1} \cdot \mathrm{h}^{-1}\right)$, PAS mantida em $105 \mathrm{mmHg}, \mathrm{FC}$ de 150 bpm, extremidades frias, com ventilação mecânica, broncoespasmo leve e sem alterações hidroeletrolíticas. Após 2 horas na UTI, começou a reagir, quando foi sedado com midazolam contínuo. Com 16 horas do final da cirurgia, já sem suporte de drogas vasoativas e hemodinamicamente estável, o paciente foi extubado, recebendo alta da UTI.

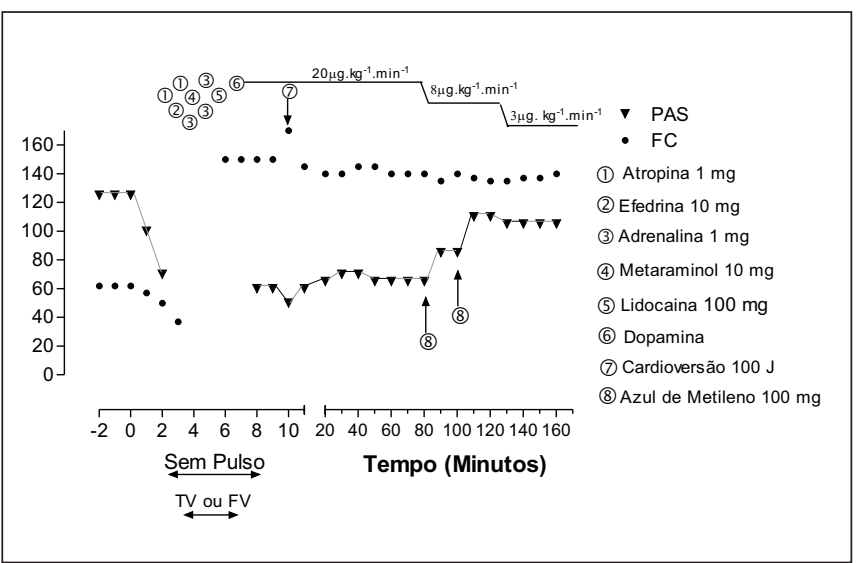

Figura 1 - Evolução da Pressão Arterial Sistólica (PAS) e Freqüência Cardíaca (FC) em relação ao Tempo de Início dos Sintomas e Medicações Utilizadas

Notar que a partir do oitavo até o octagésimo minuto o paciente apresentou intensa instabilidade hemodinâmica apesar das várias medicações utilizadas incluindo dopamina em dose de $20 \mu \mathrm{g} \cdot \mathrm{kg} \cdot \mathrm{min}^{-1}$. Após a administração da primeira dose de azul de metileno ocorreu aumento da PAS permitindo a diminuição da dose de dopamina. A segunda dose de azul de metileno também foi seguida de aumento da PAS e diminuição subseqüente da dose de dopamina para $2 \mu \mathrm{g} . \mathrm{kg} \cdot \mathrm{min}^{-1}$ que foi mantida para estimular a diurese

\section{DISCUSSÃO}

O choque anafilático, pela sua imprevisibilidade, rapidez de instalação, morbidade e mortalidade, é uma complicação temida pelos anestesiologistas. Este caso apresentou quadro clínico típico, de acordo com os sintomas mais freqüentes descritos na literatura: colapso cardiovascular $(90 \%)^{10}$, taquicardia supraventricular $(80 \%)^{11}$, parada cardíaca $(11 \%)$ ${ }^{12}$, broncoespasmo $(50 \%)^{13}$, eritema cutâneo, edema palpebral e de glote, dentre outros. Apesar da instituição imediata do tratamento clássico (adrenalina, corticosteróides, anti-histamínicos e reposição rápida de líquidos), ocorreu melhora clínica parcial, permanecendo instabilidade hemodinâmica por mais de uma hora após o início da reação. 
O papel do óxido nítrico (NO) no choque anafilático ainda é controverso e parcialmente elucidado, podendo ser responsabilizado tanto pela vasodilatação característica, quanto pela contraposição a mediadores vasoconstritores coronarianos liberados ${ }^{14}$. O uso de inibidor da NO-sintase, em cães submetidos a choque anafilático induzido, promove melhora da hipotensão arterial, mas diminui o débito cardíaco e aumenta a mortalidade ${ }^{15,16}$. Estas ações divergentes podem ser explicadas pela inibição da síntese de NO, tanto da forma induzida, quanto da forma constitutiva. O bloqueio do mecanismo constitutivo é indesejável, podendo resultar em piora do broncoespasmo e depressão cardíaca, já que NO constitutivo está envolvido em vários sistemas responsáveis pela homeostase $^{16}$.

Nas células da musculatura lisa dos vasos, o NO promove a formação de GMPc, que, em última instância, produz relaxamento e vasodilatação ${ }^{9}$. O azul de metileno promove inibição da guanilil-sintase, inibindo a formação de GMPc e bloqueando a ação vasodilatadora do NO, sem contudo agir de forma generalizada em outros tecidos e órgãos como os inibidores de NO-sintase.

Aadministração de azul de metileno por via venosa é segura, já que se trata de droga utilizada há muitos anos no tratamento de metahemoglobinemia, malária e como anti-séptico urinário. A dose letal determinada em experimentos farmacológicos in vivo em ovelhas foi maior que $40 \mathrm{mg} \cdot \mathrm{kg}^{-1} 17$. Este corante tem sido também utilizado no tratamento de estados vasoplégicos associados à sepse ${ }^{18-20} \mathrm{ou}$ em quadros vasoplégicos pós-circulação extracorpórea ${ }^{21,22}$. O uso de inibidor da NO-sintase em sepse leva à diminuição do débito cardía$\mathrm{Co}^{20}$, enquanto o uso de azul de metileno não altera ou até aumenta o débito cardíaco ${ }^{23}$, evidenciando os diferentes mecanismos de ação de cada droga. Neste caso, houve melhora significativa da PAS, imediatamente após a administração do azul de metileno.

Este relato do uso de azul de metileno, como droga coadjuvante no tratamento de choque anafilático durante anestesia, confirma sua eficácia já relatada em anafilaxia a contrastes ou antibióticos ${ }^{24-26}$.

Neste caso, o azul de metileno foi utilizado, após a terapêutica clássica para choque anafilático ter apresentado melhora clínica apenas parcial. Não se pode estabelecer de maneira definitiva a relação de causa/efeito do uso do azul de metileno e a melhora hemodinâmica, podendo ter sido conseqüência da ação tardia das drogas inicialmente utilizadas ou, simplesmente, devido à melhora espontânea com o passar do tempo. Contudo, quatro fatos sugerem que a utilização de azul de metileno tenha sido benéfica. Em primeiro lugar, a melhora hemodinâmica ocorreu sem que se tenha alterado a terapia que vinha sendo utilizada. Em segundo, o aumento de $15 \mathrm{mmHg}$ na PAS, imediatamente após a utilização da primeira dose azul de metileno. Em terceiro, o aumento de aproximadamente $20 \mathrm{mmHg}$, imediatamente após a segunda dose do corante. Por último, a possibilidade da redução da dose de dopamina de 20 para $2 \mu \mathrm{g} \cdot \mathrm{kg}^{-1} \cdot \mathrm{h}^{-1}$, mantendo-se os parâmetros hemodinâmicos.

Considerou-se que não há evidências suficientes na literatura científica que permitam utilizar o azul de metileno como droga única em anafilaxia. Portanto, até o presente momento, o tratamento clássico deve ser instituído tão logo seja possível, e o uso do azul de metileno deve ser reservado para os casos refratários, ou como droga coadjuvante. Aexperiência relatada neste caso, e em outros casos já publicados, sugere que o azul de metileno poderá vir a ter papel fundamental no tratamento de anafilaxia.

\section{Methylene Blue to Treat Anaphylaxis during Anesthesia. Case Report}

Renato Mestriner Stocche, TSA, M.D.; Luís Vicente Garcia, TSA, M.D.; Marlene Paulino dos Reis, TSA, M.D.; Jyrson Guilherme Klamt, TSA, M.D.; Paulo RobertoB. Évora, M.D.

\section{INTRODUCTION}

Anaphylaxis risk is increased during the perioperative period due to exposure to a high number of drugs. The first perioperative anaphylaxis report dates from 1936, when a patient presented unexpected reaction after the administration of d-tubocurarine ${ }^{1}$. The incidence of anesthetic allergic reactions is controversial, varying from $1 / 3,000$ to $1 / 20,000$ and, even with immediate conventional treatment (epinephrine, volume expansion, steroids, ventilatory support and anti-histaminic drugs), mortality rate may be as high as $3 \%$ and $9 \%{ }^{2-4}$.

Anaphylaxis is a complex and partially understood reaction. Several chemical mediators are released, which account for cardiovascular and respiratory collapse. Histamine and bradykinin are among major mediators ${ }^{5}$. Massive release of these substances induces nitric oxide production ${ }^{5-8}$, which increases vascular endothelium cGMP that promotes its typical arteriolar vasodilatation ${ }^{9}$.

This report describes the use of methylene blue, a cGMP-synthase inhibitor, as coadjuvant to treat anaphylaxis refractory to conventional therapy.

\section{CASE REPORT}

A 53-year-old male patient was submitted to right inguinal hernia correction under spinal anesthesia with hyperbaric 15 $\mathrm{mg}$ bupivacaine. He was given $3 \mathrm{mg}$ intravenous midazolam and monitored with cardioscopy in $\mathrm{D}_{\|}$and V5 leads, automatic blood pressure (oscillometry) and pulse oximetry. At surgery completion, patient was awakened and hemodynamically stable for more than one hour (BP 125/80 
$\mathrm{mmHg}$ ), when $1.5 \mathrm{~g}$ intravenous dipirone were given for postoperative analgesia. He immediately referred tongue tingling, evolving to bronchospasm and decreased systolic blood pressure $(70 \mathrm{mmHg})$. Oxygen $100 \%$ was administered under mask followed by $10 \mathrm{mg}$ IV ephedrine. Respiratory symptoms however have rapidly worsened with cyanosis, glottis edema, skin erythema, decreased hemoglobin saturation, blood pressure undetected by oscillometry, absence of peripheral pulses and HR decrease to $37 \mathrm{bpm}$.

Resuscitation maneuvers were started with intravenous $1 \mathrm{mg}$ epinephrine, $1 \mathrm{mg}$ atropine and $100 \mathrm{mg}$ succinylcholine, followed by tracheal intubation, manual ventilation with $100 \%$ oxygen and external cardiac massage. Cardiac electric activity was reestablished with no pulse though, when additional 1 $\mathrm{mg}$ epinephrine and $1 \mathrm{~g}$ hydrocortisone were administered, still with no hemodynamic improvement.

At 8 minutes, $1 \mathrm{mg}$ additional epinephrine was administered when patient presented sustained ventricular tachycardia, corrected with $2{\mathrm{mg} . \mathrm{kg}^{-1}}^{-1}$ idocaine. Ten minutes after VT recovery, there was hemodynamic improvement (140 bpm and SBP $=60 \mathrm{mmHg})$. Aminophylline and dopamine (20 $\mu \mathrm{g} \cdot \mathrm{kg}^{-1} \cdot \mathrm{min}^{-1}$ ) were then administered.

Twenty-five minutes later, cardiac rhythm changed to atrial fibrillation with high ventricular response and SBP decreased to $50 \mathrm{mmHg}$. Arrhythmia reverted after the third cardioversion attempt $(100,150$ and $200 \mathrm{~J})$. In the meantime, vesical and nasogastric probes were inserted, central venous puncture was performed and arterial blood gases analysis and blood electrolytes dosage were required. At 80 minutes, with volume corrected with 2500 crystalloids and $500 \mathrm{~mL}$ of $4 \%$ polyamide $\left(14 \mathrm{cmH}_{2} \mathrm{OPVC}\right)$ and with no electrolytic changes, patient maintained sinusoidal tachycardia (145 bpm) and severe hypotension (SBP between 60 and $75 \mathrm{mmHg}$ ), even after high dopamine doses $\left(20 \mu \mathrm{g} \cdot \mathrm{kg}^{-1} \cdot \mathrm{min}^{-1}\right)$.

We then decided to administer $100 \mathrm{mg}$ IV methylene blue in slow injection (5 minutes). Immediately after administration, SBP has increased to $85 \mathrm{mmHg}$. This mild improvement was maintained for 5 minutes, when additional 100 mg methylene blue was given, with subsequent SBP increase to $105 \mathrm{mmHg}$. Right after, dopamine infusion was rapidly tapered (20 minutes) from 20 to $10,7,5$ and finally 2 $\mu \mathrm{g} \cdot \mathrm{kg}^{-1} \cdot \mathrm{min}^{-1}$, which was maintained to stimulate diuresis.

Patient was transferred to the ICU 155 minutes after beginning of symptoms receiving dopamine $\left(2 \mu \mathrm{g} \cdot \mathrm{kg}^{-1} \cdot \mathrm{min}^{-1}\right)$, presenting satisfactory diuresis $\left(2 \mathrm{~mL} \cdot \mathrm{kg}^{-1} \cdot \mathrm{h}^{-1}\right), \mathrm{SBP}=105$ $\mathrm{mmHg}, \mathrm{HR}=150 \mathrm{bpm}$, cold extremities, mechanical ventilation, mild bronchospasm and with no electrolytic changes. Patient started reacting 2 hours later, when continuous IV midazolam was started. He was extubated 16 hours after surgery completion, hemodynamically stable with no vasoactive drug support, being then discharged from the ICU.

\section{DISCUSSION}

Anaphylaxis is a feared complication due to its fast onset and unexpected nature, with a high morbidity and mortality rates. This report has described a typical clinical situation, accord-

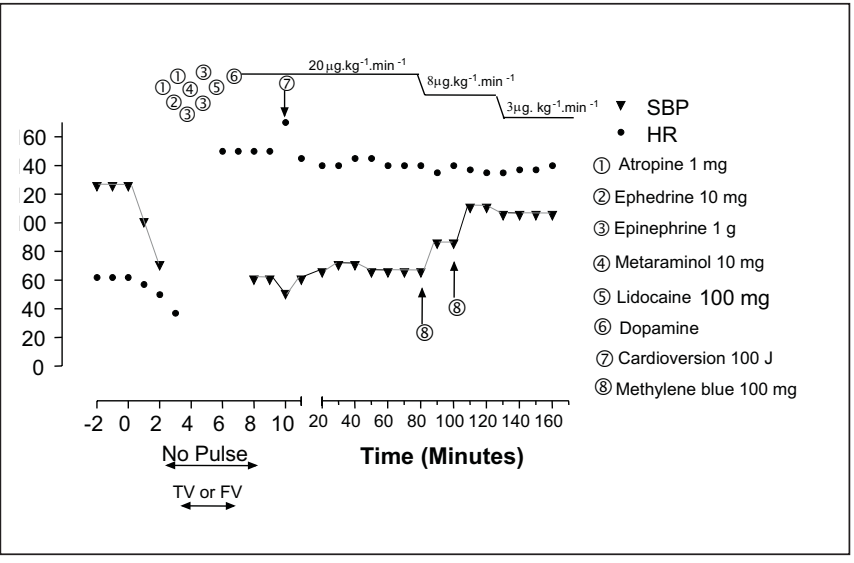

Figure 1 - Evolution of Systolic Blood Pressure (SBP) and Heart Rate as a function of Onset of Symptoms and Drugs Administered

Note that as from the $8^{\text {th }}$ through the $80^{\text {th }}$ minute patient presented severe hemodynamic instability in spite of different drugs, including $20 \mu \mathrm{g} \cdot \mathrm{kg}^{-1} \cdot \mathrm{min}^{-1}$ dopamine. After the first methylene blue dose there has been SBP increase, which has allowed for dopamine dose tapering. The second methylene blue dose was also followed by SBP increase and subsequent dopamine dose decrease to $2 \mu \mathrm{g} \cdot \mathrm{kg}^{-1} \cdot \mathrm{min}^{-1}$, which was maintained to stimulate diuresis

ing to most frequent symptoms described in the literature: cardiovascular collapse $(90 \%)^{10}$, supraventricular tachycardia $(80 \%)^{11}$, cardiac arrest $(11 \%)^{12}$, bronchospasm $(50 \%)^{13}$, skin erythema and edema (eyelid and glottis), among others. In spite of the prompt institution of conventional treatment (epinephrine, steroids, anti-histaminic drugs and rapid fluid replacement), there has been partial clinical improvement with hemodynamic instability for more than one hour after beginning of reaction.

The role of nitric oxide (NO) in anaphylaxis is still controversial and partially explained. It may be responsible for both typical vasodilatation and contraposition to released coronary vasoconstrictor mediators ${ }^{14}$. NO-synthase inhibitors in dogs submitted to induced anaphylaxis has promoted hypotension improvement but has also decreased cardiac output and increased mortality ${ }^{15,16}$. These divergent actions may be explained by NO synthesis inhibition, both in its induced and constitutive form. Constitutive mechanism inhibition is undesirable and may result in bronchospasm and more cardiac depression, since constitutive NO is involved in several systems responsible for homeostasis ${ }^{16}$.

In vessels' smooth muscle cells, NO stimulates cGMP synthesis which ultimately produces relaxation and vasodilation $^{9}$. Methylene blue inhibits guanylyl-synthase, thus reducing cGMP formation and blocking NO vasodilating action, not acting though in other tissues and organs, like other NO-synthase inhibitors do.

Intravenous methylene blue is safe; it is a drug used for many years to treat metahemoblobinemia and malaria, and as an urinary antiseptic. Lethal dose determined by in vivo pharmacological studies in ewes was higher than $40 \mathrm{mg} . \mathrm{kg}^{-1}$ 17 . This stain has also been used to treat vasoplegic states associated to sepsis ${ }^{18-20}$ or post cardiopulmonary bypass Vol. 54, Nº 6, Novembro - Dezembro, 2004 
vasoplegy ${ }^{21,22}$. The use of NO-inhibitor during sepsis leads to decreased cardiac output ${ }^{20}$ while methylene blue does not change or even increases cardiac output ${ }^{23}$, evidencing the different action mechanisms of each drug. In our case, there was a significant SBP improvement immediately after methylene blue administration.

This report on methylene blue as coadjuvant to treat anaphylaxis during anesthesia confirms its already reported efficacy in anaphylaxis to contrasts or antibiotics ${ }^{24-26}$.

In this case, methylene blue was used after obtaining only partial anaphylaxis improvement with conventional therapy. It is not possible to definitely establish the cause/effect relationship between methylene blue and hemodynamic improvement, which could have been a consequence of late effects of drugs initially used or simply spontaneous improvement with time. However, some facts suggest that methylene blue has been beneficial. First of all, hemodynamic improvement was observed without any change in the therapy already in course. Secondly, there has been a $15 \mathrm{mmHg}$ SBP increase immediately after the first methylene blue dose and an approximately $20 \mathrm{mmHg}$ increase right after the second dose. Lastly, dopamine infusion rate could be tapered from 20 to $2 \mu \mathrm{g} . \mathrm{kg}^{-1} \cdot \mathrm{h}^{-1}$ still maintaining hemodynamic parameters. We consider that there are not enough evidences in the literature to support the use of methylene blue as single therapeutic drug for anaphylaxis. So, conventional treatment should be started as soon as possible, and methylene blue should be kept for refractory cases or as coadjuvant drug. Our experience, reported in this case, and other published cases, suggest that in the future, methylene blue may play a critical role in the treatment of anaphylaxis.

\section{REFERÊNCIAS - REFERENCES}

01. Alam M, Anrep GV, Barsoum GS et al - Liberation of histamine from the skeletal muscle by curare. J Physiol, 1939;95:148-158.

02. Boston Collaborative Drug Surveillance Program. Drug induced anaphylaxis. J Am Med Assoc,1973;224:613-615.

03. Fisher MM, Baldo BA - Anaphylactoid reactions during anaesthesia. Clin Anaesth, 1984;3:677-692.

04. McKinnon RP, Wildsmith JA- Histaminoid reactions in anaesthesia. Br J Anaesth, 1995;74:217-228.

05. Babe KS, Serafin WE - Histamine, Bradykinin, and their Antagonist. Pharmacological Basis of Therapeutics, $9^{\text {th }}$ Ed, McGrall Hill Companies, USA, 1996.

06. Mitsuhata H, Shimizu R, Yokoyama MM - Role o nitric oxide in anaphylactic shock. J Clin Immunol, 1995;15:277-283.

07. Osada S, Ichiki H, Oku H et al - Participation of nitric oxide in mouse anaphylactic hypotension. Eur J Pharmacol, 1994;252:347-350.

08. Mitsuhata H, Saitoh J, Takeuchi $\mathrm{H}$ et al - Production of nitric oxide in anaphylaxis in rabbits. Shock, 1994;2:381-384.

09. Rapoport RM, Murad F - Agonist-induced-endothelium dependent relaxation in rat thoracic aorta may be mediated through cGMP. Cir Res, 1983;52:352-357.

10. Fisher MM, Baldo BA - The incidence and clinical features of anaphylactic reactions during anesthesia in Australia. Ann Franc Anesth Reanim, 1993;12:97-104.
11. Fisher MM - Clinical observations on the pathophysiology and treatment of anaphylactic cardiovascular collapse. Anaesth Intensive Care, 1986;14:17-21.

12. Laxenaire MC, Moneret-Vautrin DA, Boileau S et al - Adverse reactions to intravenous agents in anaesthesia in France. Klin Wochenschr, 1982;60:1006-1009.

13. Fisher MM, Baldo BA - Acute anaphylactic reactions. Med J Aust, 1988;149:34-38.

14. Abend $Y$, Ashkenazy $Y$, Witzling $V$ et al - Nitric oxide: a mediator in anaphylactic shock in guinea-pigs. J Basic Clin Physiol Pharmacol, 1996; 7:57-69.

15. Mitsuhata $\mathrm{H}$, Takeuchi $\mathrm{H}$, Saitoh J et al - An inhibitor of nitric oxide, $\mathrm{N}$ omega-nitro-L-arginine-methyl ester, attenuates hypotension but does not improve cardiac depression in anaphylaxix in dogs. Shock, 1995;3:447-453.

16. Mitsuhata H, Saitoh J, Hasome $\mathrm{N}$ et al - Nitric oxide synthase inhibition is detrimental to cardiac function and promotes bronchospasm in anaphylaxis in rabbits. Shock, 1995;4:143-148.

17. Burrows GE - Methylene blue: effects and disposition in sheep. J Vet Pharmacol Ther, 1984;7:225-231.

18. Marczin N, Tekeres M, Salzman AL et al -. Methylene blue infusion in septic shock. Crit Care Med, 1995;23:1936-1938.

19. Preiser JC, Lejeune P, Roman A et al - Methylene blue administration in septic shock: a clinical trial. Crit Care Med, 1995;23:259-264.

20. Zhang H, Rogiers P, Preiser JC et al - Effects of methylene blue on oxygen availability and regional blood flow during endotoxic shock. Crit Care Med, 1995;23:1711-1721.

21. Andrade JCS, Batista Filho ML, Evora PRB et al - Utilização do azul de metileno no tratamento da síndrome vasoplégica após cirurgia cardíaca. Rev Bras Cirurg Cardiovasc, 1996;11:107-114.

22. Dagenais F, Mathieu P - Rescue therapy with methylene blue in systemic inflammatory response syndrome after cardiac surgery. Can J Cardiol, 2003;19:167-169.

23. Daemen-Gubbels CR, Groeneveld PH, Groeneveld AB et al. Methylene blue increases myocardial function in septic shock. Crit Care Med, 1995; 23:1363-1370.

24. Evora PR, Roselino $\mathrm{CH}$, Schiavetto PM - Methylene blue in anaphylatic shock. Ann Emerg Med, 1997;30:240.

25. Evora PRB, Roselino CHCD, Schiavetto PM - Utilização do azul de metileno no tratamento do choque anafilático. Uma proposiçao clínica e apresentaçao de três casos. Rev Bras Terap Intens, 1997;9:126-131.

26. Evora PR, Oliveira Neto AM, Duarte NM et al - Methylene blue as treatment for contrast medium-induced anaphylaxis. J Postgrad Med, 2002;48:327.

\section{RESUMEN}

Stocche RM, Garcia LV, Reis MP, Klamt JG, Evora PRB - Uso del Azul de Metileno en el Tratamiento de Choque Anafiláctico durante Anestesia. Relato de Caso

JUSTIFICATIVA Y OBJETIVOS: En el período peri-operatorio, el riesgo de anafilaxis siempre debe ser considerado. La incidencia de reacciones alérgicas en anestesia es polémica, variando entre $1 / 3000$ a $1 / 20.000$, con mortalidad entre $3 \%$ y $9 \%$. En este caso, se relata el uso del azul de metileno como coadyuvante al tratamiento del choque anafiláctico refractario a la terapéutica tradicional.

RELATO DEL CASO: Paciente del sexo masculino, 53 años, sometido a herniorrafia inguinal bajo raquianestesia. Al final 
del procedimiento, al recibir dipirona (1,5 g), por vía venosa, el paciente inmediatamente presentó broncoespasmo, cianosis, disminución de la $\mathrm{SpO}_{2}$ y de la PS, culminando con parada cardiorrespiratoria. Fue iniciada la reanimación cardiorrespiratoria con masaje cardíaco externo, seguida de IOT e inyección de adrenalina (1 $\mathrm{mg}$ ), atropina (1 $\mathrm{mg})$, restableciéndose FC de $150 \mathrm{lpm}$, sin embargo sin pulso palpable. Se administró más $1 \mathrm{mg}$ de adrenalina además de $1 \mathrm{~g}$ de hidrocortisona, con restablecimiento de pulso central (8 minutos). A pesar de recibir dopamina $\left(20 \mu \mathrm{g} \cdot \mathrm{kg}^{-1} \cdot \mathrm{min}^{-1}\right)$, el paciente se mantuvo hipotenso $(60 \mathrm{mmHg})$ hasta 80 minutos. Se administraron $100 \mathrm{mg}$ de azul de metileno por vía venosa, cuando hubo aumento de la PS para 85 y $105 \mathrm{mmHg}$, después de la segunda dosis. Se siguió a la disminución de la dosis de dopamina de 20 para 10, 7, $5 \mathrm{y}$, finalmente, $2 \mu \mathrm{g} \cdot \mathrm{kg}^{-1} \cdot \mathrm{min}^{-1}$.

CONCLUSIONES: La anafilaxis tiene como principal mediador la liberación de histamina, que induce la producción de óxido nítrico (NO), con consecuente aumento de guanilato ciclase que promueve vasodilatación arteriolar por aumento del GMP cíclico. El azul de metileno puede ser útil en estas situaciones, pues inhibe la guanilato ciclase y consecuentemente la vasodilatación, lo que resulta en una mejoría hemodinámica. 